\title{
The role of endophenocognitypes in cognitive performance
}

\section{Kenneth Revett}

Faculty of Informatics and Computer Science,

The British University in Egypt,

El Sherouk City, Cairo 11837, Egypt

E-mail: ken.revett@bue.edu.eg

\begin{abstract}
The endophenotype concept was introduced into the literature by Gottesman and Gould in 1973 to bridge the gap between complex multi-factorial disease processes and the underlying genetic basis for such diseases. The concept has been useful in developing a more comprehensive approach to understanding a number of disease processes through the application of extra-genetic analytical approaches. The approach focuses on aspects of disease processes that are more directly linked to measurable functionand hence will tend to be more informative with respect to disease process identification than the complex genetics which ultimately may underlie the disease process. The concept has been applied most rigorously to identifying psychopathologies such as schizophrenia. Recent work has established its successful application to a wider range of cognitive disorders such as major depression and anti-social behaviour. In the process, a considerable body of cognitive processing features has been elucidated in the context of investigating the cognitive basis for these cognitive disorders. These cognitive processing features have been termed 'endophenocognitypes.' This concept retains the scientific framework proposed by Gottesman, but emphasizes the building blocks relevant to cognitive functioning directly. The focus in this paper is to discuss why the endophenocognitypes might should be deployed in the context of modelling and the subsequent development of UIs.
\end{abstract}

Keywords: affective computing; cognitive performance support; endophenocognitype; endophenotype; man-machine interaction; user interface design.

Reference to this paper should be made as follows: Revett, K. (2013) 'The role of endophenocognitypes in cognitive performance', Int. J. Cognitive Performance Support, Vol. 1, No. 1, pp.1-10.

Biographical note: The author is an associate professor at the British University in Egypt, where he teaches a variety of subjects within the computational neuroscience and artificial intelligence domains. His research focuses on the deployment of cognitive signals for UI interactions and related topics such as game play, artificial intelligence based game engine design and EEG signal processing. $\mathrm{He}$ received his $\mathrm{PhD}$ in computational neuroscience from the University of Maryland, College Park, USA in 1999. Since that time, he has engaged in studies examining the role of the brain in person/machine interactions.

USAB 2010: HCI in Work \& Learning, Life \& Leisure, Klagenfurt University, 16.-17. September 2010.

Copyright (c) 2013 Inderscience Enterprises Ltd. 


\section{Introduction}

The purpose of this paper is to present an informal discussion of the role of the endophenocognitype should play in the design decisions and development processes when in user interface design. The endophenotype in the context of UI has not been fully discussed in the literature - and this author believes that it should be given due consideration in the context of cognitive performance via user interaction. More specifically, I am referring to the inclusion of subjects with cognitive disorders - such as major depression, ADHDand related disorders (see Table 1). The argument proceeds as follows:

- upwards of $25 \%$ of the U.S. population has undergone at least one episode of affective distress in their lifetime,

- a variety of affective disorders yield significant and measurable alterations in their cognitive performance,

- many of these alterations in cognitive performance may impact how they interact with cognitively demanding processes such as user interfacesand

- $\quad$ since many aspects of daily life require such UI interactions, a significant portion of the population at large may be placed at a disadvantage if UIs don't take these factors into account.

Of course, this stream of reasoning is not new and forms the backbone of the cognitive science informed human computer interaction literature Boring (2002), Hammond, et al.

Table 1 Summary statistics for a selected subset of mental disorders obtained from the National Institute of Mental Health, applied to the 2004 census statistics (see [9]).

\begin{tabular}{|c|c|c|c|}
\hline General Classification & $\begin{array}{c}\text { DSM_IV } \\
\text { Classification }\end{array}$ & $\begin{array}{l}\text { Number in millions } \\
\text { (\% population) }\end{array}$ & $\begin{array}{c}\text { Age of onset in years } \\
\text { and median age }\end{array}$ \\
\hline $\begin{array}{l}\text { Major Depressive } \\
\text { Disorder }\end{array}$ & $292 .\{2 . .36\}$ & $14.8(6.7 \%)$ & $\geq 18$, median 32 yrs \\
\hline Dysthymic Disorder & 300.4 & $3.3(1.5 \%)$ & $\geq 18$, Median 31 yrs \\
\hline Bipolar Disorder & $296 .\{0 . .89\}$ & $5.7(2.6 \%)$ & $\geq 18$, median 25 yrs \\
\hline Schizophrenia & $295 .\{10 . .90\}$ & $2.4(1.1 \%)$ & $\begin{array}{l}\geq 18 \text {, median men: } 10- \\
24 \text {, women: } 22-32 \text { yrs }\end{array}$ \\
\hline Anxiety Disorders & 293.84 & $40.0(18.1 \%)$ & $\geq 18$, median 25 yrs \\
\hline $\begin{array}{l}\text { Obsessive-Compulsive } \\
\text { Disorder }\end{array}$ & 300.3 & $2.2(1 \%)$ & $\geq 18$, median 19 yrs \\
\hline $\begin{array}{l}\text { Generalized Anxiety } \\
\text { Disorder }\end{array}$ & 300.02 & $6.8(3.1 \%)$ & $\geq 18$, median 31 \\
\hline $\begin{array}{l}\text { Attention Deficit } \\
\text { Hyperactivity Disorder }\end{array}$ & $314 .\{00 . .90\}$ & $8.9(4.1 \%)$ & $\geq 18$, median 7 yrs \\
\hline $\begin{array}{l}\text { Antisocial Personality } \\
\text { Disorder }\end{array}$ & 301.7 & $2.2(1 . \%)$ & $\geq 18 \mathrm{yrs}$ \\
\hline $\begin{array}{l}\text { Avoidant Personality } \\
\text { Disorder }\end{array}$ & 301.82 & $11.0(5.2 \%)$ & $\geq 18$ yrs \\
\hline
\end{tabular}

The cognitive aspects of person/UI has been studies for several decadesand most readers will no doubt be extremely familiar with the GOMS models, the Model Human Processor 
(MHP)and related cognitive architectures that relate motoric and cognitive processes into a coherent framework. I argue that these models may fall victim to the following: they may normalize performance measures and then starting from the centroid of the cluster, a hyperplane is placed by moving out from the centroid, stopping at a plane based on statistical significance or some other measure of model goodness of fit. All users beyond the hyperplane are therefore considered outliers. Further, many such models make the assumption that user performance monotonically increases up to some limit. The location of the hyperplane (and hence the number of outliers) is significantly affected by the variables in the model. As will be discussed further in this discussion, the endophenocognitype concept may enhance the efficacy of the model parameters/variables based on additional neurophysiologically relevant criteria. Further, that performance displays a positive trend is just that $-\mathrm{a}$ trend. The law of large numbers may play a part here - the general idea is to extrapolate from a relatively small sample size to the general population. If the sample does encompass users/subjects at the extremes (such as those with affective disorders) then how can one be sure the UI will function effectively for such subjects? This result is just the natural consequence of the rules of engagement in scientific endeavours. How to address these issues is still an extremely important and topical area of research within the cognitive performance domain.

One approach to addressing the effect of cognitive disorders on UI interactions is to focus on users/subjects with specific disorders in the design and testing phases of UI development. For instance, studies of Parkinson's patients have been undertaken, which focuses on how success their interaction with standard UIs is with and without training Keates and Trewin (2005), Begnum (2010). This is clearly very useful and raises general awareness about users with compromised motoric functionality. What would be helpful is to integrate this work into the general UI community. Furthermore, motoric disorders tend to vary significantly as a function of the natural progression of the disorder, age and treatment regime. The results of studies focusing solely on users/ subjects within the midst of a manifest motoric disorder are not clear cut in terms of how they can inform the larger general community (though later I discuss that this 'larger' community may not be as large as we think!). In the extremely limiting case of users/ subjects in the locked in state - extreme UI designs have been able to provide a degree of utility for such cases and form probably the far end of a spectrum of compensatory UI design issues available. The question is whether we can formulate an approach that will provide a rational framework for addressing the hugely variable community of users, while still maintaining computational efficiency. For this I turn to the topic of the endophenocognitype.

Gottesman introduced (coined) the term 'endophenotype in his 1973 paper Gottesman and Shields (1973). The term embodies the concept of disentangling multi-factorial disorders such as schizophrenia, in the context of finding a genetic basis responsible for its manifestation. Work in this domain was very equivocal and hopes of finding a specific genetic signature (an SNP for instance) have failed. Through massive research efforts, the many and varied cognitive alterations associated (and really defining) schizophrenia were discovered, prevalent within the SZ community to varying degrees. These include the inability to focus attention, pre-pulse inhibition reduction, etc. What Gottesman proposed was to move the search up from the genetic level - and see if a biological basis could be found to account for these higher order cognitive alterations. He argued that since these markers may be more directly associated with higher order specific events (i.e. pulse-pair inhibition), then with the 
full disease itself, he claimed that these intermediate phenotypes should be emphasized in trying to ascertain the genetic basis of complex multi-gene based diseases such as SZ. These markers form signatures, a set of genetic minutiae (as in fingerprints), that when pieced together, yield a diagnosis.

It is noteworthy that the endophenotype concept has been refocused in the context of the 'endophenocognitype' - to reflect that the search is specific to cognitive/affective alterations. There is no reason to limit endophenotypes to cognitive processes - and in fact, the concept is applicable to all aspects of the triumvirate of motoric, sensory and cognitive schemata, unifying all 3 components if required. But the endophenocognitype specifically refers to features that derive directly from the application of endophenotypes to cognitive/ affective disorders. The claim in this discussion is that it is these very specific alterations in cognitive and/or affective capacity associated with a wide variety of affective disorders (of the DSM-IV TR type) that need to be addressed in cognitive models of UI design. These alterations in function (defining a new functional range) include working memory, timed choice-reactions, resistance to distractions, mood (euthymic vs. dsythemia), attention levels, decision making skills, etc. Gottesman and Gould (2003), De Geus and Boomsma (2001), Freedman (2003). These and certainly more yet to be discovered cognitive alterations will have an impact on how a user interacts with a system that presents a cognitive challenge. The difficulty in accommodating these issues in UI design is fully appreciated. Should a more general universal model be developed that incorporates these affective/cognitive features into its design, producing a universal UI? Should the UI be adaptive to the skills and cognitive/affective state of the user, which may vary over time, natural disorder progression, medication, or any combination of the above? These are very difficult yet extremely interesting questions that we would like to address in the International Journal of Cognitive Performance Support.

There is an estimated prevalence of mental disorders of over $26 \%$ in the US - that is, approximately 1 in 4 Americans suffer from a diagnosable mental disorder in a given year (2004 statistics). This translates into over 57 million subjects. Further, almost half of these subjects (45\%) meet criteria for 2 or more disorders Reeves et al. (2011). The categorisation of disorders mentioned above are derived from the DSM-IV classification scheme. According to this diagnostic criterion, a general summary of the prevalence of a variety of mental disorders is presented in Table 1.

In addition to the general DSM-IV categories listed in Table 1, which is only a small fraction of the available categories, U.S. national health surveys collect statistics regarding 'mentally unhealthy days.' This general measure of mental health status is typically acquired during a health-related quality of life (HRQOL questionnaire), which acquires information regarding the physical, mental, emotional and social aspects of life and summarises the mental and physical state of an individual. One of the elements in the HRQOL relates to a question regarding the number of days (in the past 30 days) when the subject felt stressed, depressive and/or exhibited emotional problems. If a subject reported $\geq 14$ such days, they are classified/defined as have frequent mental distress. The results from this appearing simple question have significant acceptable criterion validity and test-retest reliability. For a comprehensive review of this interesting and relevant topic, please consult Reeves et al. (2011).

The data in Table 1 are somewhat outdated, in that they reflect the 2004 census in the U.S. If one assumes the trend has remained the same and adjust for the increase in population (from 220 to 320 million), the number of subjects influenced by a mental disorder is 
extremely significant. If one makes the assumption that the incidence of mental disorders has remained the same, then 1 in 4 persons ( 80 million) will be classified by DSM-IV or ICD-10 as having some form of mental and/or affective disorder (in the U.S. alone). What makes matters somewhat more complicated is that the penetration is biased and therefore one can't assume that if we take $n * 4$ subjects for a study, where $n$ is some large integer, we can somehow incorporate these effects. For a discussion of the distribution of mental and/ or affective disorders, please consult Reeves et al. (2011). Given that a significant fraction of the U.S. population (not discounting the rest of the world!) is considered by modern medical and psychological health organisations to be out of the range of normalcy, how does this impact a user/subject interacting with a UI? To illustrate the potential utility of the endophenocognitype in UI technology, a set of three selected cognitive disorders (as defined by DSM-IV) is presented below.

\section{Case studies}

\subsection{Obsessive compulsive disorder}

Obsessive Compulsive Disorder (OCD) is a heritable neuropsychiatric disorder with a lifetime prevalence of 2-3\% Menzies et al. (2007). It can be characterized along two dimensions: obsessions, unwanted intrusive thoughts and actions that are typically focused on contamination, checking, or symmetry and compulsions, which can be thought of as the actions in lieu of obsessions (i.e. washing one's hands repeatedly). The neurological/ psychological basis for OCD appears to be related to a lack of inhibitory (motor) control. OCD subjects typically will perform outside of normal limits in inhibitory control as demonstrated by the results of a stop signal task Menzies et al. (2007), Chamberlain et al. (2005). The basic result of these types of studies is that OCD subjects are not able to respond quickly to inhibitory signals that occur in their environment - they tend to continue whatever action they were undertaking in the presence of a signal indicating the contrary should occur. More generally, OCD subjects tend to become regimented in their behaviour patterns. This may have a significant impact on user performance if they are exposed to different UIs that start off in a fairly similar manner, but subsequently diverge in their form and requirements from the user. One could hypothesize that an OCD user will tend to continue down a particular path that they have become accustomed to following and then fail to response to subtle cues in the environment (UI) that indicates that another path should be followed in order to complete the required task. Alternatively, an OCD user may simply find a path that they feel most comfortable with at the start of interacting with the application, which will reduce the exploration of the UI considerably.

\subsection{Major depression disorder (MDD)}

Depression, a general feeling of a lack of enthusiasm for life, is a major mood disorder that affects a vast majority of people within their lifetime (approximately $15 \%$ of the U.S. population). The major symptoms associated with (MDD) include: altered responses to stress, impaired cognitive abilities and dysfunctional ward-related behaviours Hasler et al. (2004). More specifically, subjects display a mood biased towards negativity, anhedonia, characterized by a lack of interest and reactivity to features in their environment, impairments in short and long term memory, impaired executive cognitive function, which includes 
planning, strategy selection and monitoring performance, psychomotor changes and a magnification of responses to normal stressors. Clearly, a seriously depressed individual will typically not engage as effectively with their environment (i.e. a UI) as a non-depressed individual. There is a considerable amount of experimental results which have produced biological endophenotypes, based on alterations in REM sleep, abnormalities in brain structure and function, altered psychopharmacology studies and intra-signalling changes have been proposed as endophenotypes for MDD. It is interesting to note that many of these biological endophenotypes have a direct cognitive impact and hence should be labelled enophenocognitypes to be more precise.

\subsection{Attention-deficit hyperactivity disorder (ADHD)}

ADHD has been popularized in recent years probably because of its apparent increase in prevalence amongst children. It has been given a variety of labels such as: attention deficit disorder (ADD), hyperactivity, minimal brain dysfunction and minimal brain damage to name some of the more popular terms, which by the way are utilised by the medical community Robbins et al. (2011), Castellanos and Tannock (2002). The definition utilised in this discussion focuses on that described in Table 1 (from the DSM-IV) definition. Accordingly, the primary features that form part of an operational definition include the following: locomotor hyperactivity, deficiency in response inhibition (as measured via the stop-signal task), shortened delay gradient (measured as the effectiveness of a reward as a function of delay time), temporal processing (lapses in focus and concentration) and a reduction in visual-spatial working memory function. Clearly, all but locomotor hyperactivity is cognitive in effect and hence they should be classified as endophenocognitypes. One would expect that one or more of these defining characteristics of ADHD could impact the ability of a user to interact effectively in a highly complex and demanding UI.

\subsection{Endophenocognitypes, neuroscience and UI}

These are only a sample of the list of disorders contained within the DSM-IV and ICD-10 (albeit with a slightly different classification criteria), selected because they highlight cognitive impairments that may directly impact the person's ability to interact with complex machinery/UIs effectively. For a fuller expose on the role of endophenotypes in this context, see De Geus and Boomsma (2001). Further, the number of potentially affected users is very large - approximately $25 \%$ of the U.S. population has or will undergo at least one episode within their lifetime and many will present with multiple disorders. This discussion is not suggesting that we build UIs with the ability to support a schizophrenic at the height of their disorder, but rather, that we should include subjects that are considered by DSM-IV/ ICD-10 standards as having one or more cognitive disorders to be subjects when designing and testing UIs. The question revolves around the interplay between UI technologies (HCI generally) and neuroscience Gardner (1987).

Boring has argued that HCI per se could form a unifying platform that would assist in the structuring of cognitive neuroscience into a coherent and well defined discipline Boring (2002). This is an interesting perspective - and a challenge, requiring the close collaboration of both communities. The endophenocognitype perspective provides a quantitative approach to this potential collaboration, providing quantitative measures of 
specific cognitive abilities. The issue in the context of endophenocognitypes that should be addressed is how to incorporate this knowledge into UI design specifications. Clearly, we do not require subjects to be scanned prior to being able to use an UI effectively. Also, we do not need to design a UI that will be useable equally effectively by a person under the grips of full blown schizophrenia and a normal control. The point made here is that the hyperplane that is drawn around subjects within a population should be drawn in lieu of subject selection criteria that incorporate the large number of potential subjects that with DSM-IV labels. This may require performing UI tests that include subjects with specific mental disorders in sufficient numbers to truly test how well they perform. There is a significant body of published information could be called upon when devising UIs - and should be incorporated into cognitive models which many UIs are designed around. This in turn should lead to the deployment of appropriate testing conditions that would not treat users with disorders as outliers, but as bonified members of the user community.

\section{Conclusion}

I would like to end this discussion by examining how the role of the endophenocognitype could be applied to the papers included in this inaugural issue. In the paper by (Nap et al. (2013), see this volume) discusses the role of self-efficacy, which can be influenced by many factors such as experience and cognitive ability. In terms of cognitive ability, the paper stresses that age tends to reduce levels of self-efficacy, which in turn places demands on the UI such that they should proffer positive reinforcement whenever possible to enhance self-efficacy and reduce stress induced UI interactions. Clearly, age per se tends to reduce sensory/motor responses, which in turn mat require moving the hyperplane for senior citizens outwards. Furthermore, there is a significant increase in the development of more serious cognitive deficits with age, such as Alzheimer's and other forms of dementias. These deficits are gradual in their course of effect and must be addressed, as this paper nicely discussed (in the specific context of age), in a fuller context that includes the effects of the enhanced likelihood of significant cognitive deficits that accompany the ageing process.

The role of self-efficacy and aging has also been addressed in the context of manmachine interaction was investigated in the context of using domestic appliances (see Glasgow and Higgins (2013) in this volume). The authors state that using basic home products may present difficulties in the ageing population, as reaction times, binding graphical elements to physical actions and general lack of sensory acuity may hamper effective interactions. A mentioned previously, the effect of ageing may produce in addition to a general reduction in sensory processing abilities, it is also accompanied by an increase in cognitive impairments, which coupled together, provide significant challenges to the design of UIs.

Computer-assisted rehabilitation is a very interesting topic (see Nicolau et al. (2013) in this volume), which focuses on how trained professionals will be able to utilise IT to assist in patient rehabilitation. The results of this study indicate that having external feedback in the rehabilitation sessions significantly assisted the physical therapists in their work and also provided valuable and positive effects to the patients as well. In this scenario, we have an opportunity to examine how an interaction between 2 or more people, within a common 
framework, may interact with one another. Typically, most UIs are designed around a solo user - and the issue of group interaction is not included in the design. More specifically, if an UI was designed for single user use, how would it fair when it was used in a multi-user fashion?

The role of pauses in Man-Machine Interactions was investigated (see Ghazarian (2013) in this volume), to determine how pauses in MMI can be interpreted with respect to learning complex behaviours such as a computer program. The question addressed is whether pauses can be used as a measure of skill level, in the performance of a specific task. And further, whether pauses can be used as a general method for estimating the level of difficulty of learning a particular MMI. The results from this study indicate that there is a correlation between number of pauses and skill level. It would be interesting to examine this question with ADHD subjects, where focus and concentration might play a factor in their ability to acquire the necessary skill s to become an expert user.

In a study deploying augmented reality (see Turner et al. (2013) in this volume), users were exposed to a minimalist AR application in order to determine if users filled in the blanks, thereby reducing the computational overhead of producing a full-blown and realistic VR simulation environment. Users were expected to fill in the gaps using the information provided and context information (i.e. a tourist scenario). This type of UI is very interesting in the context of endophenocognitypes, as it relies almost solely on the users' interaction with the world in a strictly cognitive context (developing internal models of the world). Users with cognitive disorders might be expected to respond quite differently, along several axes, relative to normal controls.

Lastly, in a paper which investigated he combined use of EEG and NIR to monitor subjects when undertaking mathematical and concentration based challenges (see Gupta and Ramaswamy (2013) in this volume), in the context of a BCI scenario. The use of EEG and NIR are what the neuroscience community utilises for acquiring endophenocognitypes data and in addition, BCI type applications as described in this paper are purely cognitive UIs. Though this study utilised a relatively small number of subjects, it would be very instructive to utilise subjects from different populations to see how they respond. This is the essence of the endophenocognitype approach.

\section{Call for papers (CFP)}

The editorial board at the ICJPS is encouraging submissions of papers for the next issue to focus on the issue of measuring cognitive performance, from a wide range of subjects in terms of cognitive disorders. The submissions can focus on:

- Methodological strategies (i.e. EEG, NIR, GSR, etc. based measures)

- Direct comparison of control and subjects with known cognitive disorders

- Cognitive models incorporating affective/cognitive disorders in the context of MMI/ UI generally

- Endopheno(cogni)types from a neuroscience perspective

- Other topics related to the question of how cognitive capacities influence learning in all forms

- Othergeneral topics related to biological markers, endophenotypes or endophenocognitypes 
- Cognitive science and HCI

- $\quad$ Neuroscience and UI generally.

\section{Acknowledgement}

We would like to acknowledge the contributions from participants of the USAB 2010: HCI in Work \& Learning, Life \& Leisure (all aspects of Human-Computer Interaction and Usability) co-located with the Alps Adria Psychology Conference 2010 Klagenfurt University, 16-17 September 2010 http://usab2010.uni-klu.ac.at/.

\section{References}

Begnum, M.E.N. (2010) 'Challenges for Norwegian PC-users with Parkinson's disease - a survey', K.Miesenberger et al. (eds.), ICCHP 2010, Part I, LNCS 6179, pp.292-299.

Boring, R.L. (2002) 'Human-computer interaction as cognitive science', Proceedings of the Human Factors and Ergonomics Society, $46^{\text {th }}$ Annual Meeting, pp.1767-17771,

Castellanos, F.X. and Tannock, R.M. (2002) 'Neuroscience of attention-deficit/hyperactivity disorder: the search for endophenotypes', Nature Reviews, Neuroscience Vol. 3, pp.617-628.

Chamberlain, S.R., Blackwell, A.D., Fineberg, N.A., Robbins, T.W. and Sahakian, B.J. (2005) 'The neuropsychology of obsessive compulsive disorder: the importance of failures in cognitive and behavioural inhibition as candidate endophenotypic markers', Neuroscience and Biobehavioral Reviews, pp.1-21.

De Geus, E.J.C. and Boomsma, D.I. (2001) 'A genetic neuroscience approach to human cognition', European Psychologist, Vol. 6, No. 4., pp.241-253.

Freedman, R. (2003) 'Electrophysiological phenotypes', Methods Molecular Medicine, Vol.77, pp.215-225.

Gardner, H. (1987) 'The Mind's New Science: A History of the Cognitive Revolution', New York, NY: Basic Books.

Ghazarian, A. (2013) 'Pauses in man-machine interactions: a clue to user's skill levels', International Journal of Cognitive Performance Support, Vol. 1, No. 1.

Glasgow, A. and Higgins, P. (2013) 'Design of domestic appliances for the cognitively impaired', International Journal of Cognitive Performance Support, Vol. 1, No. 1.

Gottesman, I.I. and Gould, T.D. (2003) 'The endophenotype concept in psychiatry: etymology and strategic intentions', Am J. Psychiatry, Vol. 160, No. 4, pp.636-6445.

Gottesman, I.I. and Shields, J. (2001) 'Genetic theorizing and schizophrenia', Br. J. Psychiatry, Vol. 122, pp.15-30.

Gupta, C.N. and Ramaswamy, P. (2013) 'using EEG and NIRS for brain-computer interface and cognitive performance monitoring: a pilot study', International Journal of Cognitive Performance Support, Vol. 1, No. 1.

Hammond, N., Gardner, M.M., Christie, B. and Marshall, C. (1987) 'The role of cognitive psychology in user-interface design', In M.M. Gardiner and B. Christie (eds.), Applying Cognitive Psychology to User-Interface Design (pp.13-54), Chichester, UK: John Wiley \& Sons.

Hasler, G., Drevets, W.C., Manji, H.K. and Charney, D.S. (2004) 'Discovering endophenotypes for major depression', Neuropsychopharmacology Vol. 29, pp.1765-1781.

Keates, S. and Trewin, S. (2005) 'Effect of age and Parkinson's Disease on cursor positioning using a mouse', Proceedings of the ASSETS,05 Conference, October 9-12, 2005, Baltimore, MD, USA, pp.68-75.

Menzies, L., Achard, S., Chamberlain, S.R., Fineberg, N., Chen-C-H., del campo, N., Sahakian, B.J., Robbins, T.W. and Bullmore, E. (2007) 'Neurocognitive endophenotypes of obsessivecompulsive disorder', Brain Vol. 130, pp.3223-3236. 
Nap, H.H., de greef, H.P. and Bouwhuis, D.G. (2013) 'Self-efficacy support in senior computer interaction', International Journal of Cognitive Performance Support, Vol. 1, No. 1.

Nicolau, H., Guerreiro, T., Pereira, R., Goncalves, D. and Jorge, J. (2013) 'Computer assisted rehabilitation: towards effective evaluation', International Journal of Cognitive Performance Support, Vol. 1, No. 1.

Reeves, W.C., Strine, T.W., pratt, L.A., Thompson, W., Ahluwalia, I., Dhingra, S.S., McKnight-Eily, L.R., Harrison, L., D’angelo, D.V., Williams, L., Morrow, B., Gould, D. and Safran, M.A. (2011) 'mental illness surveillance among adults in the United States, Centers for Disease Control and prevention', Morbidity and Mortality Weekly Report (MMWR), suppl. Vol. 60, September 2.

Robbins, T.W., Gillan, C.M., Smith, D.G., de Wit, S. and Ersche, K.D. (2011) 'Neurocognitive endophenotypes of impulsivity and compulsivity: towards dimensional psychiatry, Special issue: Cognition in Neuropsychiatric disorders', Trends in Cognitive Sciences, Vol. 6, No. 1, pp.81-91.

Turner, P., Turner, S. and Burrows, L. (2013) 'Creating a sense of place with a deliberately constrained virtual environment', International Journal of Cognitive Performance Support, Vol. 1, No. 1. 